\title{
AS MEDIDAS APLICADAS AOS ACORDOS SPS E TBT SOBRE AS EXPORTAÇÕES BRASILEIRAS DE FUMO
}

\section{MEASURES APPLIED TO AGREEMENTS SPS AND TBT ON BRAZILIAN EXPORTS OF TOBACCO}

\author{
Carlos Otávio de Freitas \\ Universidade Federal de Viçosa - Viçosa - MG - Brasil \\ Marília Fernandes Maciel Gomes \\ Universidade Federal de Viçosa - Viçosa - MG - Brasil \\ Fernanda Maria de Almeida \\ Universidade Federal de Viçosa - Viçosa - MG - Brasil \\ Fernanda Aparecida Silva \\ Universidade Federal de Viçosa - Viçosa - MG - Brasil
}

\begin{abstract}
Resumo: O presente estudo tem como objetivo analisar os efeitos das medidas não tarifárias, especificamente as medidas sanitárias e fitossanitárias - SPS e medidas técnicas - TBT, sobre as exportações brasileiras de fumo e se tais efeitos permaneceram nos períodos subsequentes à emissão das notificações. Para tal, foi estimado um modelo gravitacional utilizando uma amostra de 89 países importadores do fumo do Brasil entre o período de 1997 a 2011 . O método utilizado na estimação das equações foi o modelo Poisson-Pseudo Maximum Likehood-PPML. Os resultados obtidos mostram que as medidas ou SPS afetaram negativamente o fluxo internacional do comércio de fumo. Além disso, verificou-se que esses efeitos perduraram nos períodos posteriores à emissão da notificação, sendo que as empresas exportadores podem demorar até dois anos para se ajustarem às exigências requeridas. Dentre os objetivos das notificações SPS, a maior parte está relacionada a medidas para proteção da saúde humana e proteção da planta, o que pode explicar a maior dificuldade de adequação, pois são exigências que demandam grandes investimentos. Já as medidas TBT não foram estatisticamente significativas, confirmando o caráter não restritivo dessas notificações sobre o comércio internacional de fumo. Apesar da maior parte das notificações ao fumo estarem associadas a medidas técnicas, os resultados indicaram que elas não configuraram como barreiras ao comércio de fumo. Isso pode sugerir que as exigências a elas referidas, que, em sua grande maioria, foi referente à
\end{abstract}


rotulagem e/ou embalagem, foram atendidas pelo Brasil sem maiores dificuldades. Dessa forma, torna-se importante a inclusão de questões referentes à regulamentação dessas medidas nas discussões e processos de tomada de decisão das empresas exportadoras de fumo. Em termos de ações governamentais, seria interessante a criação ou aperfeiçoamento de políticas no sentido de aumentar o caráter informativo das medidas sanitárias, principalmente as que envolvem questões relacionadas à saúde humana e proteção da planta, de forma a reduzir o tempo que as empresas exportadoras utilizam para se adequar a tais exigências.

Palavras-Chave: Fumicultura. Medidas não tarifárias. Efeito residual. Modelo gravitacional. PPML.

Abstract: The aim of this study is to analyze the effect of non-tariff measures, specifically the Sanitary and Phytosanitary - SPS and Technical Barriers to Trade - TBT, on brazilian exports of tobacco and if this effect remained in the periods following the issuance of notifications. For that a gravity model was estimated using a sample of 89 importer's brazilian tobacco between 1997 to 2011. The method used in the estimation of equations was the model Poisson-Pseudo Maximum Likehood (PPML). The results show that the sanitary and phytosanitary measures (SPS) affect the international flow of trade in tobacco negatively. Furthermore, was verified that this effect persisted during the periods after the issuance notification, and exporting companies can take up to 2 years to adjust the requirements set. Among the objectives of SPS notifications, most are related to measures for the protection of human health and protection of the plant, which may explain the greater difficulty of adaptation, since they are requirements that require large investments. The technical measures (TBT) were not statically significant, confirming a non-tariff such notifications on international trade in tobacco. Although most of the notifications to tobacco are associated with technical measures, the results indicated that they have not configured as barriers to trade in tobacco. This may suggest that the requirements referred to them, which was mostly related to labeling and / or packaging, were attended by Brazil without difficulty. Thus, it becomes important to include questions concerning the regulation of these measures in the discussions and decision-making processes of companies exporting tobacco. In terms of government actions, it would be interesting to establishment or enhancement of policies to increase the informative character of health measures, particularly those involving issues related to human health and plant protection, in order to reduce the time that exporters use to conform to such requirements.

Key words: Tobacco. Non-tariffs measures. Residual effect. Gravity model. PPML.

\section{INTRODUÇÃO}

A fumicultura brasileira, desde 1993, tornou-se um dos setores mais competitivos do agronegócio brasileiro, com o país assumindo a posição de segundo maior produtor e principal exportador mundial de 
fumo (SECEX, 2012). Em 2014, segundo a Associação dos Fumicultores do Brasil - Afubra (2015), foram produzidas 751 mil toneladas de fumo em folha, equivalentes a $12,4 \%$ do total mundial. No país, a produção se concentra na região Sul, que foi responsável por mais de $97 \%$ da produção nacional de fumo no ano de 2014.

Em relação ao comércio externo, o fumo destaca-se como um dos principais produtos da pauta de exportações brasileiras. Nos últimos cinco anos, cerca de $85 \%$ da produção nacional tem sido direcionada ao mercado externo, representando, em média, $2 \%$ do total de vendas brasileiras no exterior e $30 \%$ do total das exportações mundiais de tabaco. Na safra 2013/14, as exportações atingiram o volume de 627,2 mil toneladas, e o valor obtido foi de US\$ 3,2 bilhões. Esse total exportado representou um aumento de $96,2 \%$ se comparado à safra de $1995 / 96$, em que foram exportados 319,7 mil toneladas de fumo. Os principais destinos do tabaco nacional foram a União Europeia, Ásia e Estados Unidos, que absorvem cerca de 42\%, $28 \%$ e $10 \%$ das exportações, respectivamente (SINDITABACO, 2015).

Apesar de os Estados Unidos e a União Europeia terem destaque nas importações de fumo, existem barreiras comerciais que limitam a entrada do fumo brasileiro nestes países. Nos Estados Unidos, há determinação legal de percentual mínimo de $75 \%$ de fumo doméstico na produção de cigarros, além das cotas tarifárias de importação com uma tarifa extracota proibitiva de $350 \%$. Na União Europeia, por sua vez, existe uma tarifa específica entre 220 e 560 euros por tonelada de tabaco em folha, além de tributação média em torno de 20,7\%. Entretanto, por meio das negociações da Rodada do Uruguai, houve redução dessa proteção tarifária praticada pelos países nos últimos anos (SECEX, 2015).

Uma das principais consequências dessas negociações foi a reestruturação do Acordo Geral de Tarifas e Comércio-GATT como Organização Mundial de Comércio-OMC, resultando, entre outras intervenções, no compromisso de redução das tarifas para produtos industriais e agrícolas e no reforço de regras que deram origem a acordos específicos. Entre os acordos específicos, com a finalidade de coordenar e limitar o uso de Medidas Não Tarifárias-MNT ao comércio, destacam-se o acordo sobre medidas TBT e o acordo para aplicação de medidas SPS. Desse modo, os países membros comprometem-se a 
emitir uma notificação ${ }^{1}$ sempre que houver modificações nos regulamentos internos que possam afetar o fluxo do comércio (ALVES, 2008).

Segundo a OMC (2012), o acordo SPS tem como finalidade impedir que as medidas justificadas para a proteção à saúde humana, animal e vegetal constituam barreiras ao comércio. Já o TBT procura impedir que medidas técnicas como normas, regulamentos para a produção, rotulagem e procedimentos para a avaliação de conformidade atuem no mesmo sentido. Além disso, um dos fatores que podem estar relacionados com a adoção dessas MNT é a crescente exigência dos consumidores dos países importadores com aspectos relacionados à segurança alimentar e ambiental (SILVA et. al, 2011 ).

A utilização dessas medidas pode trazer consequências positivas na economia, como a harmonização dos regulamentos das normas internacionais, o aumento da qualidade dos produtos, a segurança humana, animal e vegetal e a facilitação do fluxo de comércio. Contudo, se identificadas como barreiras, tais medidas podem provocar limitações ao comércio, aumento dos custos para adequação aos regulamentos e aumento dos preços dos produtos. Além disso, os custos envolvidos na adequação às medidas podem inviabilizar as exportações das empresas, principalmente pequenas e médias, uma vez que precisam se adequar a requisitos distintos daqueles adotados em seu próprio país. Assim, caso não consigam se ajustar a tais exigências, devido ao alto investimento necessário, o efeito dessas medidas pode perdurar por períodos subsequentes à sua emissão, inviabilizando seu acesso ao mercado externo. Dessa forma, torna-se pertinente investigar os efeitos dessas medidas sobre o comércio internacional ao longo do tempo.

Diversos autores desenvolveram trabalhos empíricos buscando analisar o impacto das medidas não tarifárias no comércio. Alves (2008), ao verificar os efeitos das MNT sobre as exportações brasileiras de carne

\footnotetext{
1 Notificação é um documento que um país membro emite, junto à OMC, com o objetivo de determinar regras sobre o comércio internacional. Nesse documento, constam informações a respeito do país notificador e da Agência Nacional responsável pela criação das referidas normas, além dos países notificados, dos produtos atingidos, das normas a serem adotadas e dos objetivos que justificam sua legitimidade.
} 
de frango, identificou alto grau de proteção nos mercados da União Europeia, envolvendo muitas linhas tarifárias e alto valor das exportações sujeitas à imposição dessas barreiras. Swann et al. (1996) verificaram o efeito das normas sobre o desempenho comercial da Inglaterra, concluindo que a força relativa do país em termos de normas melhora o seu balanço comercial, mas também pode tornar o mercado mais aberto. Além desses, outros estudos desenvolvidos por Schlueter e Wieck (2009), Burnquist e Souza (2010), Disdier, Fontagné e Mimouni (2008), Otsuki, Wilson e Sewadeh (2001) e Fassarella, Souza e Burnquist (2011) também analisaram os efeitos de MNT sobre o comércio.

Dado o exposto, o presente trabalho visa a verificar o efeito das MNT, ao longo do tempo, sobre as exportações brasileiras de fumo. Especificamente, pretende-se analisar a evolução das medidas não tarifárias no setor mundial de fumo e dos seus efeitos, de modo a verificar se têm sido facilitadoras ou barreiras ao comércio.

Nesse sentido, estudos que avaliam as restrições impostas, na forma de medidas não tarifárias sobre o fumo para exportação, são relevantes, dada a grande importância do setor no que se refere à geração de empregos e divisas para o país, ao crescimento da produção e das exportações e à importância assumida pelo Brasil no mercado internacional nos últimos anos.

Este artigo está estruturado em cinco seções, além desta introdução. Na seção dois, é exposto o referencial teórico utilizado; na seção três, são apresentadas a metodologia e a fonte de dados do trabalho; na seção cinco, os dados são analisados e discutidos; por fim, na seção seis, são expostas as principais conclusões do trabalho.

\section{REFERENCIAL TEÓRICO}

O referencial teórico utilizado no presente artigo é composto por duas partes. Primeiramente é apresentada a teoria das barreiras regulatórias ao comércio internacional, especificamente as teorias apresentadas por Thilmany e Barret (1997) e Roberts, Orden e Josling (1999). A seguir, a teoria referente ao modelo gravitacional é descrita.

\subsection{Teoria das barreiras regulatórias ao comércio internacional}


As medidas sanitárias e técnicas são utilizadas com a finalidade de proteger a vida e a saúde humana, animal e vegetal, além de preservar o meio ambiente. Contudo, essas regulamentações também podem funcionar como uma forma de protecionismo quando não existem fundamentos que as justifiquem, com o intuito, por exemplo, de proteger produtores domésticos. Dessa forma, a imposição de uma regulamentação pode ter impactos positivos e/ou negativos sobre os preços, a produção e o comércio e dependem da natureza informativa das notificações SPS e TBT (THILMANY; BARRET, 1997).

Teorias desenvolvidas que buscam verificar o impacto dessas regulamentações mostram a dificuldade de identificar ex-ante os efeitos líquidos para as economias, uma vez que a imposição de uma regulamentação por um país pode gerar consequências opostas para o fluxo internacional de comércio.

Thilmany e Barret (1997) mostram, na Figura 1, a complexidade da análise dos efeitos das regulamentações sanitárias e técnicas.

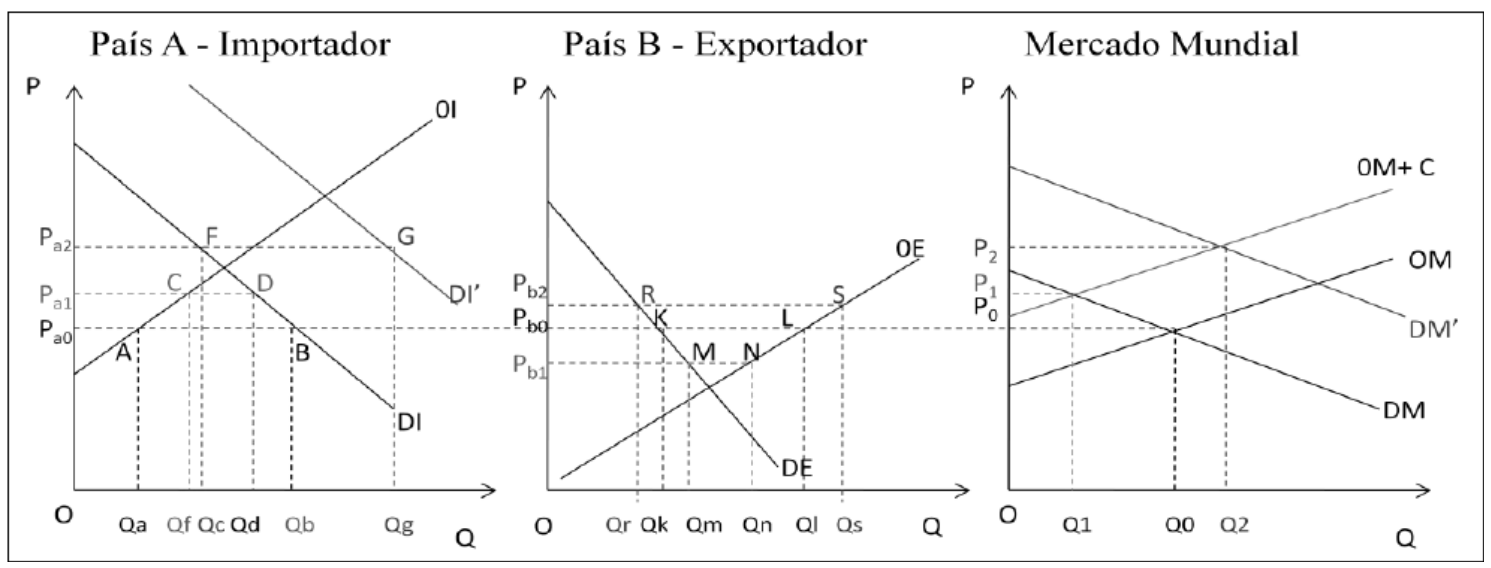

Figura 1. Efeitos de barreiras regulatórias; deslocamento da curva de excesso de oferta e de demanda.

Fonte: Thilmany e Barret (1997).

Analisando um cenário de livre comércio, em que o preço de equilíbrio inicial é $\mathrm{Po}$, o país $\mathrm{A}$ importa o volume $(\mathrm{Qb}-\mathrm{Qa})^{2}$ do país exportador, que gera um excedente correspondente a $(\mathrm{Q} I-\mathrm{Qk})$. No mercado mundial, terceiro gráfico, a curva OM representa o excedente

\footnotetext{
2 Os símbolos $Q$ referem-se aos níveis de quantidade apresentados na Figura 1.
} 
produtivo ofertado, dado que os preços internacionais são superiores ao preço de equilíbrio do país exportador. Do mesmo modo, a curva DM (referente à demanda do mercado mundial de fumo) ilustra o excesso de demanda por fumo, dada a diferença entre a quantidade demandada e a ofertada no mercado nacional, quando o preço de equilíbrio do país $\mathrm{A}$ é maior que os preços internacionais.

Supondo que ocorra a utilização de um instrumento regulatório não tarifário pelo país importador, o preço mais elevado ( $\mathrm{Pa} 1)$ no país $\mathrm{A}$ faz com que a demanda de fumo por importação seja reduzida (0Qd), com concomitante estímulo à produção doméstica desse produto (0Qc). Assim, a quantidade importada (Qd - Qf) e o bem-estar agregado (região $A B C D$ ) no país $A$ são reduzidos quando comparados ao cenário de livre comércio. A curva de excesso de oferta no mercado mundial desloca-se para cima e para a esquerda $(\mathrm{OM}$ para $\mathrm{OM}+\mathrm{C})$, em que $\mathrm{C}$ representa o custo de adequação das empresas devido às novas exigências. Dessa forma, um novo equilíbrio é estabelecido, gerando uma redução do volume comercializado de $0 Q$ o para $0 Q 1$ e um aumento nos preços internacionais de fumo de Po para $\mathrm{P} 1$.

Considerando agora que as empresas importadoras (consumidores) recebam informações sobre as exigências e seus benefícios relacionados à segurança e qualidade do fumo, ocorrendo um deslocamento da curva de demanda DI para DI' no país A e DM para DM' no mercado mundial, com o novo preço internacional (Pa2), a quantidade exportada de fumo pelo país B (Brasil) é aumentada (Qs - Qr) na mesma proporção que a quantidade de importação demandada pelo país i (Qg - Qc), e o bem-estar agregado melhora nos dois países.

Contudo, a introdução de um regulamento informativo também pode gerar um efeito negativo sobre o comércio, se o aumento na demanda interna do país importador não for suficiente para compensar o aumento dos custos das empresas exportadoras de fumo, decorrente da adequação da nova exigência. Nesse caso, o volume comercializado sob livre comércio (0Q0) pode ser superior ao volume de equilíbrio resultante (0Q2).

Assim, antecipar os efeitos de uma nova exigência pode ser complexo, já que dependem da sua natureza (não informativa ou informativa). Caso a regulamentação seja informativa, os resultados ainda estarão sujeitos à forma como os consumidores assimilarão a 
informação. Desse modo, os efeitos sobre o bem-estar dos consumidores e dos produtores, tanto no país importador, como no exportador, dependerão da amplitude do deslocamento da curva de demanda, o qual será reflexo das escolhas dos indivíduos na presença das medidas regulatórias (THILMANY; BARRET, 1997).

A análise apresentada por Roberts, Orden e Josling (1999) aborda a imposição do regulamento de forma semelhante à teoria explicada por Thilmany e Barret (1997), descrita anteriormente na análise da Figura 1. Entretanto, Roberts, Orden e Josling (1999) utilizam o conceito de externalidades para abordar o problema. A externalidade negativa decorre do fato de os consumidores não terem informações suficientes sobre o produto importado. De acordo com o Roberts, Orden e Josling (1999), essa abordagem pode ser aplicada para interpretar os efeitos de medidas sanitárias e técnicas, como atributos de qualidade e/ou regime de rotulagem obrigatória. A questão fundamental nesse modelo é determinar se os benefícios auferidos pelo consumidor, a partir da informação contida no regulamento, compensam o custo de adequação das empresas exportadoras a esse regulamento (Figura 2).

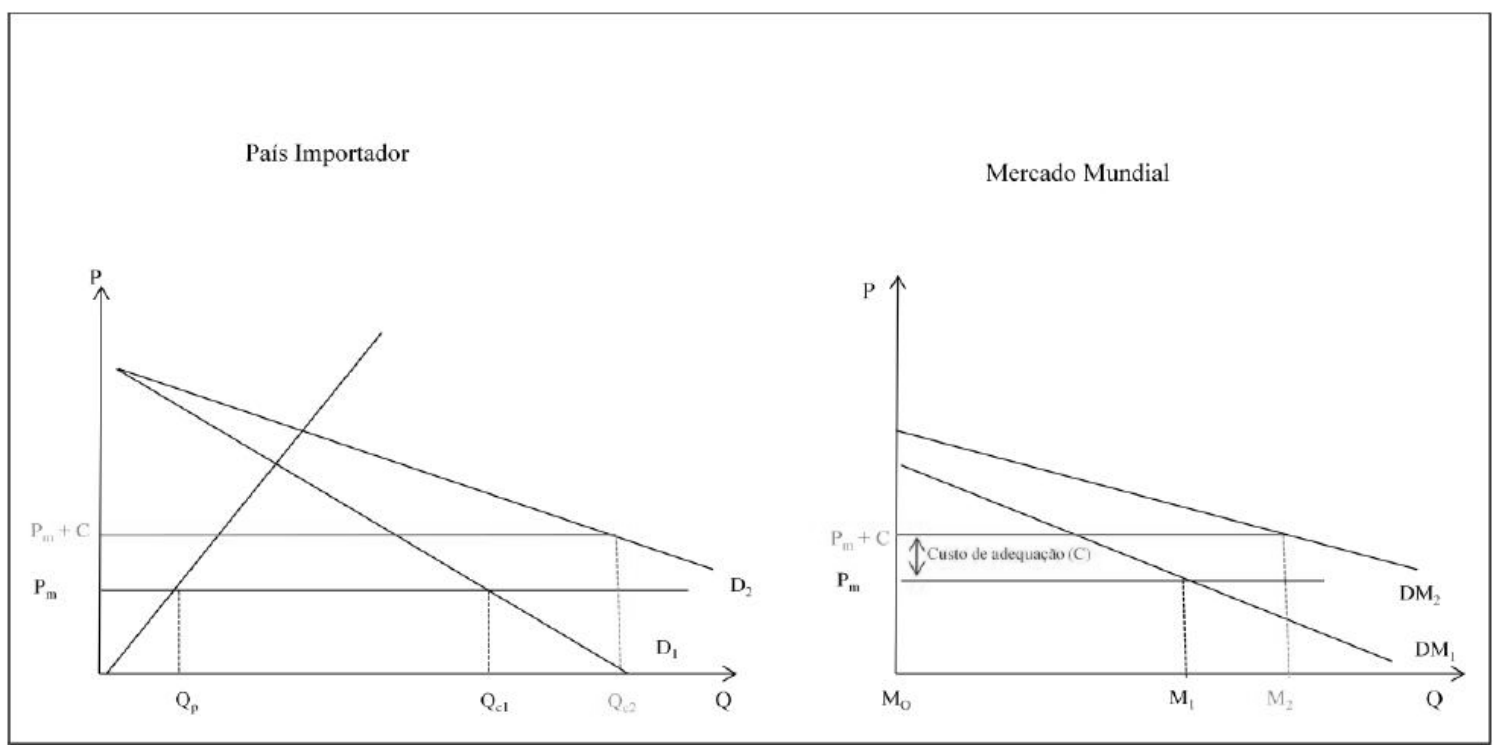

Figura 2. Efeito das barreiras regulatórias; deslocamento da curva de excesso de demanda.

Fonte: Roberts, Josling e Orden (1999). 
O primeiro diagrama representa o mercado doméstico, formado pela curva de demanda (D1) e de oferta $(0)^{3}$. O mercado internacional é apresentado no segundo diagrama, em que a curva DM1 representa a diferença entre a quantidade demandada e a ofertada no mercado doméstico. Sob condições de livre comércio, o preço mundial é Pm. A esse preço, há um excesso de demanda no mercado doméstico, em que a quantidade produzida é Qp e a quantidade consumida é Qc1; logo, o país importa o volume $(\mathrm{Qc} 1-\mathrm{Qp})$, que, por sua vez, é equivalente a (M1 - M0). Assim, define-se o ponto de equilíbrio em (Pm, M1).

A utilização da medida não tarifária desloca a curva de demanda doméstica para cima e para a direita (D1 para D2), consequentemente, a curva de demanda mundial passa de DM1 para DM2. Esse deslocamento pressupõe que a imposição do regulamento aumente a confiança dos consumidores do país quanto à qualidade do produto, o que reduz a externalidade associada à ausência de informação. Contudo, esse regulamento também eleva o preço mundial de $\mathrm{Pm}$ para $\mathrm{Pm}+\mathrm{C}$, em que $C$ é o custo de adequação à nova exigência. Esse novo cenário implica aumento do volume comercializado no mercado internacional de fumo para $(\mathrm{M} 2-\mathrm{M} 0)>(\mathrm{M} 1$ - M0), dado pelo incremento da quantidade consumida no mercado doméstico para Qc2 > Qc1.

No cenário apresentado na Figura 2, a regulamentação aumentou a confiança dos consumidores, deslocando a demanda para a direita, a ponto de compensar os custos de adequação. Entretanto, segundo os autores, se a regulamentação não proporcionar informações adicionais que permitam aos consumidores elevar significativamente a demanda, o custo de adequação será maior que o deslocamento da demanda, reduzindo, deste modo, a quantidade comercializada no mercado internacional de fumo em folha.

Desse modo, as teorias de Thilmany e Barret (1997) e de Roberts, Orden e Josling (1999) mostram que os efeitos da medida não tarifária dependem da reação dos consumidores frente ao caráter informativo do regulamento. Entretanto, como a magnitude dessa reação não é conhecida a priori, o resultado da introdução do regulamento sobre o comércio pode ser ambíguo. Assim, prever os efeitos das medidas

\footnotetext{
3 Para maiores detalhes sobre a análise do equilíbrio do mercado doméstico, bem como sobre detalhes acerca das curvas de demanda e oferta, ver Pindyck e Rubinfeld (2005).
} 
técnicas e sanitárias no comércio internacional de produtos agrícolas, como o fumo, neste estudo, pode ser difícil, pois, como analisado, os resultados estão sujeitos à forma como os consumidores reagem na presença de tais medidas.

\subsection{Modelo Gravitacional}

O modelo gravitacional vem sendo bastante difundido em diferentes trabalhos relacionados com o comércio internacional. Sua aplicabilidade deriva da física e se baseia na premissa de que "quanto maior a renda e a população de um parceiro comercial e quanto menor a distância em relação a ele, maior o montante de comércio entre duas nações. Uma das principais razões para a difusão desse procedimento é o eficiente ajuste que ele tem proporcionado em muitos trabalhos empíricos" (DA MATA; FREITAS, 2008).

A estrutura teórica e a consistência do modelo gravitacional remetem a diversas contribuições, cujas características comuns

podem se fundamentar na completa especialização de produtos, segundo Anderson (1979), Bergstrand $(1985,1989)$, Helpman e Krugman (1985), Deardoff (1998) e Anderson e VanWincoop (2003;2004), bem como na extrapolação do conceito de vantagens comparativas, quando atritos de comércio como custos de transporte e outras barreiras comerciais são considerados, permitindo, assim, avaliações dos seus efeitos sobre as negociações entre países.

O modelo é empregado de forma intensiva para a análise de políticas domésticas, influenciando a competitividade internacional. Diversos autores introduzem normas e regulamentações domésticas como proxies para analisar esses impactos, empregando o modelo gravitacional. Entre eles, destacam-se Otsuki et al. (2001), Disdier, Fontagné e Mimouni (2008) e Chen, Yang e Findlay (2008), que enfocam os efeitos da regulamentação aplicada a alimentos, produtos processados e não processados sobre o comércio dos países.

As contribuições teóricas sobre o modelo gravitacional foram recentemente reforçadas por Anderson e van Wincoop (2003, 2004). No estudo, esse modelo é derivado de funções de oferta e demanda para países exportadores e importadores em condições de equilíbrio geral. Esse modelo considera um sistema de demanda para o qual se 
pressupõe uma função de utilidade de elasticidade de substituição constante CES (Constante elasticity of substitution). A equação gravitacional é definida por

$$
X_{i, t, t}=\frac{E_{j, t} Y_{i, t}}{Y_{t}}\left(\frac{C_{i j, t}}{P_{j, t} \Pi_{i, t}}\right)^{\left(1-\sigma_{k}\right)}
$$

em que $X_{i j, t}$ são as exportações de fumo em folha do Brasil para o país j no período $\mathrm{t} ; E_{j, t}$, o dispêndio da economia j; $Y_{i, t}$, o Produto Interno Bruto-PIB do país importador $\mathrm{j} ; Y_{t}$, a produção agregada mundial; $\sigma_{k}$, a elasticidade de substituição; $C_{i j, t}$, o custo de comércio incorrido pelos exportadores para o país importador j; e $P_{j, t} \Pi_{i, t}$ representam os índices de preços para o país exportador i (no caso Brasil) e do país importador j.

A importância da contribuição desses autores é amplamente reconhecida na literatura, ainda que não tenha sido empregada com frequência em análises aplicadas pela sua relativa complexidade operacional (FEENSTRA, 2004).

Contribuições posteriores, entre as quais se destaca o desenvolvimento de Feenstra (2004), mostram que a inclusão de efeitos fixos específicos para o controle da heterogeneidade não observável entre países gera os mesmos resultados obtidos por Anderson de van Wincoop (2003), sem perda significativa de eficiência.

\section{REFERENCIAL ANALÍTICO}

O referencial analítico utilizado para identificar os efeitos das barreiras não tarifárias sobre as exportações brasileiras de fumo baseiase na teoria gravitacional, apresentada anteriormente. Especificamente, foram estimadas duas equações gravitacionais por meio do método PPML sendo os procedimentos utilizados apresentados detalhadamente a seguir.

\subsection{Modelo de Análise}


Antes de apresentar o modelo empírico utilizado neste trabalho, deve-se definir o termo que expressa os custos de comércio que afetam as exportações do setor $k$ do país j para o país i $\left(C_{i j, t}\right)$. Segundo Cheng e Wall (2005), a utilização de efeitos fixos para os pares de países pode captar os custos de comércio não observados diretamente, tais como os custos de transporte, particularidades geográficas e fatores históricos. Assim, o procedimento para o controle desses efeitos define custos de comercialização que interferem no comércio bilateral como uma função de variáveis específicas a cada economia. A equação 4 relaciona o custo de comércio com as tarifas médias aplicadas, com as medidas sanitárias e fitossanitárias e as medidas técnicas.

$$
\operatorname{Ln}\left(C_{i, t}\right)=\beta_{3} \operatorname{Ln}\left(1+\tau_{i}\right)+\beta_{4}\left(\operatorname{SPS}_{i, t}\right)+\beta_{7}\left(T B T_{i, t}\right)
$$

Assim, obtém-se a especificação econométrica utilizada para verificar o impacto das notificações SPS e TBT na exportação do fumo brasileiro:

$$
\begin{aligned}
& \operatorname{Ln}\left(M_{i, t}\right)=\alpha_{i j}+\gamma_{t}+\beta_{1} \operatorname{Ln}\left(Y_{i, t}\right)+\beta_{2} \operatorname{Ln}\left(Y_{j, t}\right)+\beta_{3} \operatorname{Ln}\left(1+\tau_{i, t}\right)+\beta_{4}\left(S P S_{i, t}\right) \\
& +\beta_{5}\left(\operatorname{SPS} S_{i, t+1}\right)+\beta_{6}\left(\operatorname{SPS} S_{i, t+2}\right)+\beta_{7}\left(T B T_{i, t}\right)+\beta_{8}\left(T B T_{i, t+1}\right)+\beta_{9}\left(T B T_{i, t+2}\right) \\
& +\beta_{10} \operatorname{Ln}\left(\operatorname{Dist}_{i j}\right)+\beta_{11} \text { Front }_{i j}+\mathcal{E}_{i, t}
\end{aligned}
$$

em que $M_{i j, t}$ representa as importações de fumo pelo país i do país j no ano $\mathrm{t} ; \mathrm{t}=1997$ a 2011 ; $\alpha_{i j}$ representa os efeitos fixos para os pares de países; $\gamma_{t}$ representa variáveis binárias para os anos da amostra; $Y_{i . t}$ e $Y_{j . t}$ são o PIB per capita do país importador i e exportador $\mathrm{j}$; $\tau_{\bar{i}}$ representa a tarifa média aplicada pelo país importador; $S P S_{i . t}$, dummy, que recebe o valor um se houver pelo menos uma notificação SPS do país importador no ano t; $T B T_{i . t}$, dummy que recebe o valor um se houver pelo menos uma notificação TBT do país importador no ano t; Dist $t_{i, j}$ representa a distância geográfica entre os países; Front $t_{i j}$, dummy que assume $o$ valor de um se os dois países fizerem fronteira; e $\varepsilon_{i j . t}$ representa o erro aleatório. 
A equação 3 foi estimada utilizando dados em painel, considerando os efeitos fixos bilaterais, dado que o objetivo é captar o impacto de variáveis específicas a cada economia (como, por exemplo, a presença de regulamentos sanitários ou técnicos) sobre o fluxo de comércio bilateral. Para captar esses efeitos, são criadas variáveis dummies para os fluxos de comércio entre pares de países, de forma a possibilitar a expressão de diferenças por meio do intercepto, que varia entre os países.

Para captar o efeito das notificações nos períodos subsequentes à sua emissão, ou seja, para verificar o tempo de adequação das medidas não tarifárias, foram utilizadas as dummies $S P S_{i . t+1}, S P S_{i . t+2}, T B T_{i . t+1} \mathrm{e}$ $T B T_{\text {i.t+2 }}$. As variáveis recebem o valor um para cada um dos dois períodos subsequentes à emissão da notificação $(t+1$ e $t+2)$ e zero, caso contrário4.

Quando se trata de mensurar o efeito de barreiras comerciais, o número de observações nulas tende a ser importante e sua ignorância pode enviesar os coeficientes estimados. Uma das alternativas existentes para tomar em conta a informação contida nos fluxos nulos é o método PPML ${ }^{5}$, proposto por Santos Silva e Tenreyro (2006), utilizado neste trabalho. Esse método é considerado adequado, pois suas estimativas são consistentes na presença de heterocedasticidade e de fluxos bilaterais nulos ${ }^{6}$ ou não informados na base de dados (SANTOS SILVA; TENREYRO, 2006). Nesse caso, a equação 3 é expressa na forma multiplicativa, seguindo uma função exponencial com a variável dependente expressa em nível, conforme demonstrado nos trabalhos de Santos Silva e Tenreyro (2006) e Siliverstovs e Schumacher (2007).

Um ponto a ser destacado na abordagem empírica se refere à definição dos fluxos comerciais. Apesar de o modelo teórico estar

\footnotetext{
${ }^{4}$ Houve casos em que foram emitidas mais de uma notificação por ano, entretanto, este estudo não considerou o número de notificações por período. Independentemente de ter havido uma única notificação ou três, por exemplo, as dummies para medidas TBT ou SPS receberam o valor unitário.

5 Para mais detalhes e informações sobre o modelo de regressão Poisson e suas propriedades, ver Gourieroux, Monfort e Trognon (1984) e Santos Silva e Tenreyro (2006).

6 Os fluxos comerciais nulos são decorrentes de arredondamento, erros de informação ou simplesmente inexistência de comércio (SANTOS SILVA; TENREYRO, 2006).
} 
definido em termos das exportações do país i para o j $\left(X_{i j, t}\right)$, grande parte dos trabalhos empíricos utiliza como variável dependente as importações do país i oriundas do país $\mathrm{j}\left(M_{i j, t}\right)$. Uma das razões para isso é que os dados de importação dos países tendem a ser de melhor qualidade que os dados de exportação (BALDWIN; TAGLIONI, 2006).

\section{2 Fontes de Dados}

Os dados utilizados referentes ao PIB dos países selecionados, para o período de 1997 a 2011 , foram obtidos no site do Banco Mundid. Os dados sobre importação foram obtidos na Commodity Trade Statistics Data Base-COMTRADE. Quanto à variável distância, foi obtida do banco de dados do Centre D'Études Prospectives et d'Informations Internationales-CEPII (2012).

Foram selecionados os 89 principais países importadores do fumo brasileiro em 2011, que absorveram cerca de 99\% do total exportado pelo Brasil. Alguns países não foram incluídos pela indisponibilidade de alguns dados ${ }^{7}$, contudo, tais países têm pequena participação no comércio internacional, absorvendo menos de $1 \%$ do fumo brasileiro exportado.

A coleta das notificações, tanto do sistema SPS quanto TBT, foi feita no site da OMC. Para isso, foram coletadas as notificações utilizando o Sistema Harmonizado-SH de dois e quatro dígitos. Quanto aos dados referentes à tarifa de importação, foram obtidos no banco de dados do Market Access Map- MACMAP.

\section{RESULTADOS E DISCUSSÃO}

Antes de analisar os resultados do modelo econométrico estimado, é apresentada uma análise das notificações emitidas no período analisado.

7 Os países não incluídos foram Sérvia, Iraque, Belize, Tonga, Seychelles, Sri Lanka, Zimbabwe, Montenegro, Maurícia, Rep. da Moldávia. 


\subsection{Análise geral das notificações}

Para esta pesquisa, foram coletadas as medidas emitidas à OMC, considerando o sistema SH de dois e quatro dígitos no período de 1997 a 2011 , totalizando 89 notificações, sendo que as medidas técnicas somaram 62 e as sanitárias 27.

A Figura 3 apresenta a evolução do total das notificações mundiais aos acordos TBT e SPS emitidas para o fumo no período de análise. Observa-se que a tendência geral foi de crescimento, havendo maior aumento a partir de 2001. Um fato que pode estar associado a esse comportamento é o início do processo de elaboração da Convenção-Quadro para o controle do $\mathrm{Tabaco}^{8}$, que ocorreu durante a $52^{\text {a }}$ Assembleia Mundial de Saúde em 1999, em que os países membros da Organização Mundial de Saúde-OMS reconheceram a necessidade de criação de medidas para redução do consumo e prevenção de doenças provocadas pelo uso do tabaco. Em 2008, foi emitido o maior número de notificações, havendo queda nos anos seguintes, com o ano de 2010 representando o menor número de emissões.

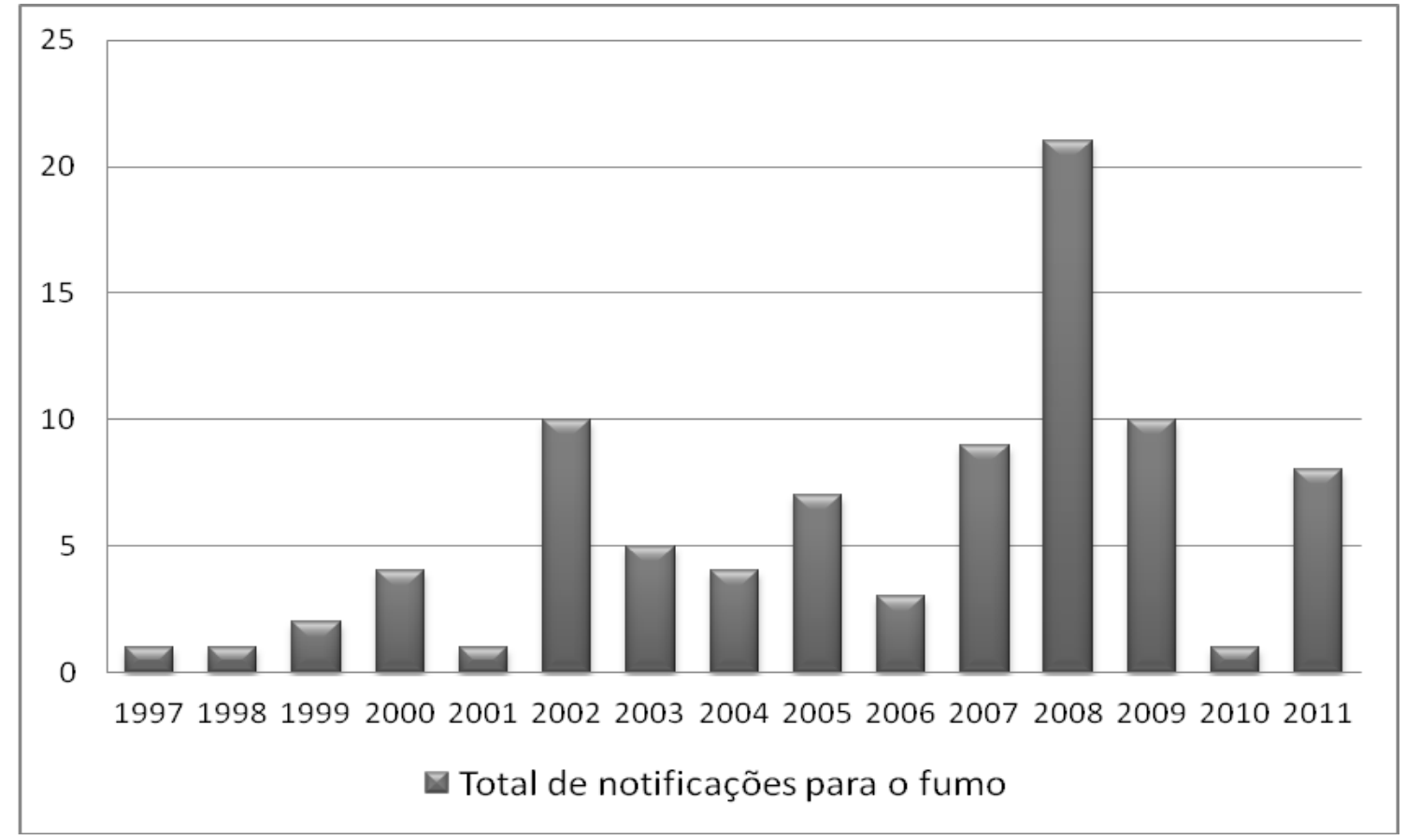

8 A Convenção-Quadro foi o primeiro tratado internacional de saúde pública da história. Este tratado fixa padrões internacionais para o controle do tabaco, com providências relacionadas à propaganda e patrocínio, à política de impostos e preços, à rotulagem, ao comércio ilícito e ao tabagismo passivo, entre outras medidas. 
Figura 3. Evolução do total de notificações para o fumo de 1997 a 2011 (SH de dois e quatro dígitos).

Fonte: Dados da Pesquisa.

A evolução das importações de fumo de 1997 a 2011 é apresentada na Figura 4. Verifica-se que, no período analisado, as importações do produto brasileiro cresceram em média $7,89 \%$ ao ano. Entretanto, se analisado juntamente com a Figura 3, observa-se que as notificações SPS e TBT não acompanham a evolução das importações de forma similar. O ano de 2009 foi o de maior destaque, gerando um montante superior a US\$ 3 bilhões, porém, o total de notificações foi menos da metade do emitido em 2008. Em 2010, essa diferença é ainda maior, enquanto o valor gerado pelas importações do fumo nacional foi próximo ao de 2008, cerca de US $\$ 2,7$ bilhões, o número de notificações emitidas foi o menor de todo a série analisada.

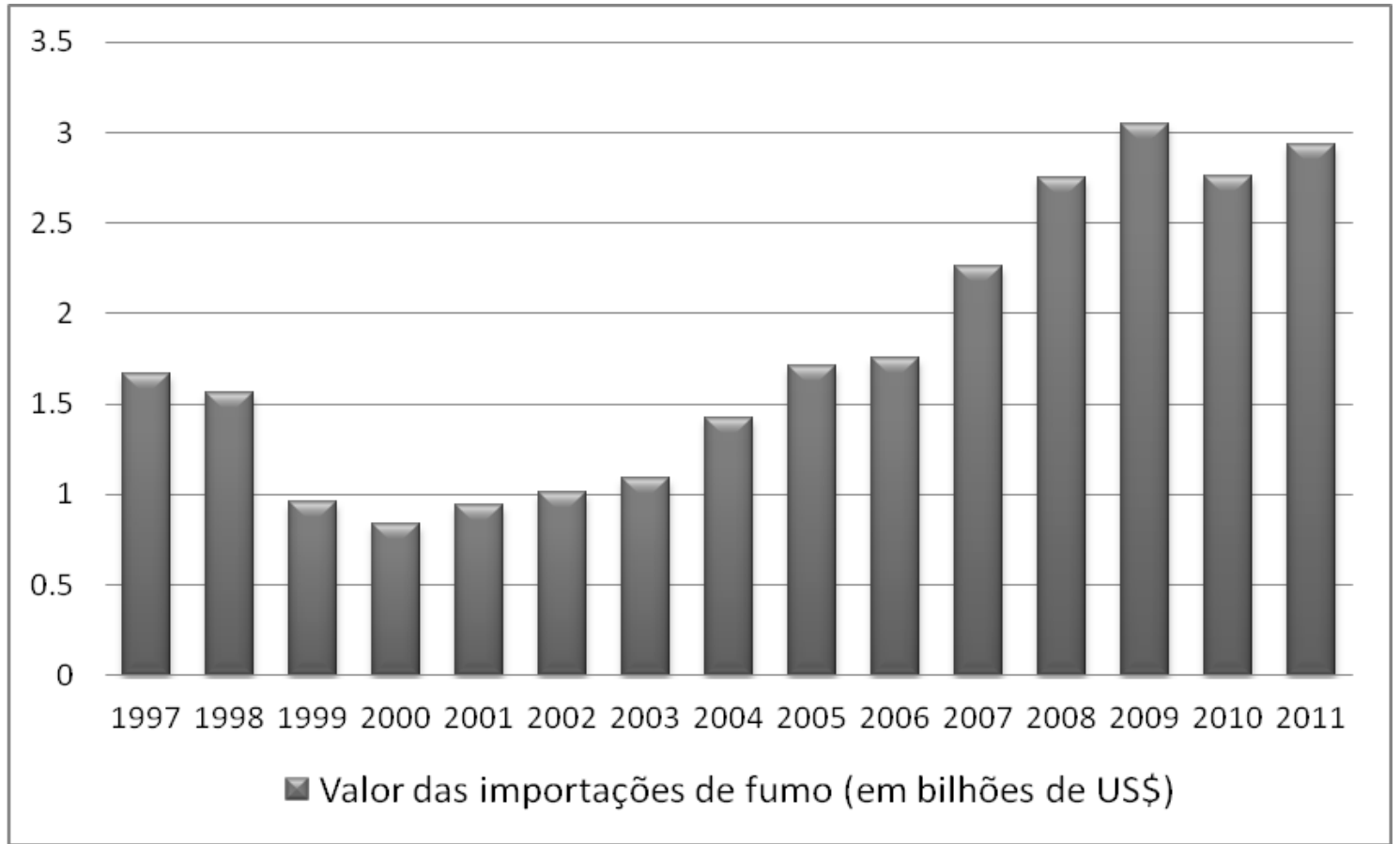

Figura 4. Evolução das importações de fumo (em bilhões de US\$) de 1997 a 2011. Fonte: Dados da Pesquisa.

Em relação à participação dos países membros da OMC na emissão das notificações, considerando as medidas SPS e TBT, observou-se que os principais foram os Estados Unidos (16\%), Tailândia 
(15\%), Guatemala (12\%), China (11\%) e Peru (9\%), sendo responsáveis, conjuntamente, por $63 \%$ do total das medidas emitidas.

A análise do modelo econométrico apresentada a seguir permite verificar se o impacto dessas medidas não tarifárias sobre o fluxo internacional do comércio de fumo foi positivo, atuando como facilitadora, ou negativo, agindo como barreira ao comércio, e se o efeito persistiu por períodos subsequentes à emissão.

\subsection{Análise do impacto das medidas sanitárias, fitossanitárias e técnicas}

$\mathrm{Na}$ Tabela 1 são apresentados os resultados das estimativas do modelo gravitacional utilizado para verificar o impacto das medidas SPS e TBT no comércio internacional do fumo brasileiro, considerando os 89 países selecionados da amostra. ${ }^{9}$

Tabela 1. Estimação do modelo gravitacional por meio do modelo PPML

\begin{tabular}{ccccc}
\hline Importação & Coeficiente & $\begin{array}{c}\text { Erro Padrão } \\
\text { Robusto }\end{array}$ & Estatística Z & P-valor \\
\hline Ln(Yi) & -0.15148 & 0.3035 & $-0.50^{\text {Ns }}$ & 0.618 \\
Ln(Yj) & 0.30093 & 0.0450 & $6.68^{*}$ & 0.000 \\
Ln(distij) & -0.63275 & 0.1012 & $-6.25^{*}$ & 0.000 \\
Ln(tarifa) & 6.18112 & 1.0315 & $5.99^{* *}$ & 0.000 \\
Frontij & 0.10622 & 0.0516 & $2.06^{* *}$ & 0.040 \\
SPSt & -0.08204 & 0.0336 & $-2.44^{* *}$ & 0.015 \\
TBTt & 0.02279 & 0.0342 & $0.67^{N S}$ & 0.505 \\
Const. & 4.25069 & 8.5669 & $0.50^{N S}$ & 0.620 \\
R $^{2}$ & 0.45325 & - & - & - \\
$\mathrm{n}^{\circ}$ observações & 1335 & - & - & - \\
\hline Fons
\end{tabular}

Fonte: Resultados da Pesquisa.

Nota:* significativo a $1 \%$; ** significativo a $5 \%$; NS - Não significativo a $10 \%$.

A partir do modelo estimado, observou-se que o coeficiente calculado para o PIB do país exportador de fumo (Lnyi), no caso o Brasil, além de estatisticamente não significativo a $10 \%$, apresentou sinal contrário ao esperado. Entretanto, de acordo com United Nations Conference on Trade and Development-UNCTAD (2008), esse resultado

${ }_{9}^{9}$ As estimativas foram feitas por meio do software STATA 12. 
pode ser justificado. Quando se estuda o comércio dos países como um todo, à medida que a renda é elevada, os países exportadores produzem maiores variedades de produtos, implicando aumento geral das exportações. Contudo, isso não acontece, necessariamente, nas análises para produtos individuais, caso do fumo neste trabalho. Incrementos no PIB do país exportador não irão gerar, necessariamente, aumento nas exportações de fumo, mas poderão ser favoráveis ao aumento do consumo interno e, principalmente, ao aumento da produção e do comércio de outros produtos, o que eleva as exportações agregadas. Resultado semelhante foi encontrado nos trabalhos de Almeida (2009) e Alves (2012).

O coeficiente estimado para o PIB dos países importadores do fumo brasileiro (Lnyj) foi estatisticamente significativo a $1 \%$ e apresentou sinal esperado, indicando que um aumento de $1 \%$ na renda desses países eleva as importações de fumo em $0,3 \%$, em média.

Em relação à variável Lndistij, utilizada como Proxy 10 dos custos de comércio, verificou-se que as importações de fumo variaram de forma inversa com a distância entre o Brasil e seus parceiros comerciais, conforme o esperado. Pelo coeficiente estimado, observou-se que um aumento em $1 \%$ na distância entre os países reduz as importações em 0,63\%, em média. Esse resultado é corroborado por Alves et al. (2011), que analisaram o impacto das medidas SPS e TBT nas importações brasileiras de uva in natura e encontraram resultados semelhantes para a variável representativa da distância entre os países.

Quanto às tarifas efetivamente aplicadas, foi utilizada a variável Lntarifa para mensurar o feito dessas barreiras tarifárias sobre as importações do fumo brasileiro. Apesar de ter sido estatisticamente significativa, apresentou um sinal contrário ao esperado, mostrando que, neste caso, um aumento na tarifa aplicada elevaria as importações de fumo. Esse resultado é coerente com o encontrado por Schlueter e Wieck (2009) e Damião (2011). Como argumentado pelos autores, acredita-se que um dos possíveis fatores que explicam esse resultado é que, no período analisado, mesmo nos anos em que o fumo foi tarifado,

10 Uma variável é denominada "proxy" quando esta é utilizada para captar o efeito de outras variáveis semelhantes. De acordo com o Gujarati (2006), proxy é uma variável que substitui aproximadamente a variável que estamos procurando. 
houve aumento do seu preço internacional e também da quantidade demandada, o que pode ter anulado o efeito negativo da tarifa aplicada.

$O$ resultado encontrado para variável Frontij, que representa a existência de fronteira entre os países, também foi significativo e apresentou sinal esperado. Nesse sentido, o sinal positivo encontrado indica relação positiva entre as importações do fumo brasileiro e o comércio com os países vizinhos.

Em relação às variáveis utilizadas para mensurar o efeito das medidas não tarifárias, representadas pelas notificações SPS e TBT, observou-se que apenas a primeira foi significativa, apresentando sinal negativo. Assim, o coeficiente estimado, que mede o impacto da presença de regulamentos sanitários e/ou fitossanitários (SPS), indicou que o comércio de fumo entre os países foi menor na presença dessas notificações. Nesse caso, o caráter restritivo dessas medidas predominou sobre o caráter informativo, restringindo o fluxo internacional do comércio de fumo. Resultado semelhante foi encontrado por Fassarella et al. (2010) ao analisar os efeitos das regulamentações sanitárias e fitossanitárias sobre o comércio das Américas. Já a variável TBT, utilizada para identificar o efeito das medidas técnicas sobre o comércio, foi não significativa, indicando que as emissões dessas notificações não afetaram o comércio de fumo no período analisado.

Por fim, foram incluídas progressivamente as variáveis SPSt +1 , $\mathrm{SPSt}+2, \mathrm{TBTt}+1$ e TBTt+2 na tentativa de identificar se o efeito das notificações permanecem em períodos posteriores à sua emissão, ou seja, para tentar capturar o efeito residual dessas medidas. O resultado obtido é apresentado na Tabela 2. 
Tabela 2. Estimação do modelo gravitacional por meio do modelo PPML, considerando o efeito residual das medidas não tarifárias

\begin{tabular}{|c|c|c|c|c|}
\hline \multirow[b]{2}{*}{ Importação } & \multicolumn{3}{|c|}{ Erro Padrão } & \multirow[b]{2}{*}{ P-valor } \\
\hline & Coeficiente & Robusto & Estatística z & \\
\hline $\operatorname{Ln}(Y i)$ & -0.16657 & 0.3087 & $-0.54 \mathrm{NS}$ & 0.590 \\
\hline $\operatorname{Ln}(Y j)$ & 0.31040 & 0.0454 & $6.84 *$ & 0.000 \\
\hline Ln(distij) & -0.65260 & 0.1020 & $-6.40 *$ & 0.000 \\
\hline Ln(tarifa) & 6.39274 & 1.0392 & $6.15^{*}$ & 0.000 \\
\hline Frontij & 0.11395 & 0.0529 & $2.15^{* *}$ & 0.031 \\
\hline SPSt & -0.09116 & 0.0339 & $-2.69 *$ & 0.007 \\
\hline SPSt+1 1 & -0.07262 & 0.0378 & $-1.92 * * *$ & 0.055 \\
\hline SPSt +2 & -0.11677 & 0.0500 & $-2.33 * *$ & 0.020 \\
\hline TBTt & 0.01785 & 0.0341 & $0.52^{\mathrm{NS}}$ & 0.600 \\
\hline $\mathrm{TBT}+\mathrm{t} 1$ & -0.02878 & 0.0440 & $-0.65^{N S}$ & 0.513 \\
\hline $\mathrm{TBT}+\mathrm{t}+2$ & -0.01864 & 0.0290 & $-0.64 \mathrm{NS}$ & 0.521 \\
\hline Const. & 4.58714 & 8.7173 & $0.53^{\mathrm{NS}}$ & 0.599 \\
\hline $\mathbf{R}^{2}$ & 0.45486 & - & - & - \\
\hline$n^{\circ}$ observações & 1335 & - & - & - \\
\hline
\end{tabular}

Fonte: Resultados da Pesquisa.

Nota:* significativo a $1 \%$; ** significativo a $5 \%$; *** significativo a $10 \%$; NS - Não significativo a $10 \%$.

A partir dos resultados do modelo estimado, verificou-se que as medidas sanitárias e/ou fitossanitárias continuaram afetando o fluxo do comércio de fumo, mesmo em períodos subsequentes à sua notificação, uma vez que os coeficientes estimados para as variáveis SPSt+1 e $\mathrm{SPSt}+2$ foram significativos com sinal negativo. Esse resultado expõe a dificuldade das empresas exportadoras de fumo para se adequarem a essas medidas, que podem implicar aumento de custo de produção e, consequentemente, elevação no preço do produto por mais de um período, dado o caráter restritivo dessas notificações. Em relação às medidas técnicas, as variáveis acrescentadas também foram não significativas, confirmando o caráter não restritivo das notificações TBT sobre o comércio internacional de fumo.

Os coeficientes estimados para as demais variáveis não sofreram alterações relevantes em relação à significância e sinais encontrados se comparadas ao modelo estimado anteriormente, seguindo, assim, a análise já descrita.

De modo geral, apesar de a maior parte das notificações ao fumo estarem associadas a medidas técnicas, os resultados indicaram que 
elas não configuraram como barreiras ao comércio de fumo. Isso pode sugerir que as exigências a elas referidas, em sua grande maioria relacionadas à rotulagem e/ou embalagem, foram atendidas pelo Brasil sem maiores dificuldades. Já os efeitos dos acordos SPS foram negativos ao comércio, sendo que as empresas exportadoras podem demorar até dois anos para se ajustar às exigências requeridas. Entre os objetivos das notificações SPS, a maior parte está relacionada a medidas para proteção da saúde humana e proteção da planta, o que pode explicar a maior dificuldade de adequação, pois são exigências que demandam grandes investimentos.

\section{CONCLUSÃO}

O presente trabalho buscou verificar o impacto das medidas SPS e TBT no comércio internacional de fumo do Brasil, sendo avaliados os 89 principais países importadores do produto. Além disso, o estudo também permitiu avaliar se o efeito de tais medidas permanece nos períodos posteriores à emissão das notificações.

Os coeficientes estimados para o modelo proposto foram estatisticamente significativos e apresentaram sinais condizentes com o mercado internacional de fumo, com exceção da variável do PIB do país exportador, ou seja, o Brasil.

Os resultados obtidos para análise das notificações indicam que as medidas sanitárias e/ou fitossanitárias impostas pelos países importadores tiveram efeito negativo sobre o comércio, atuando como barreiras ao fluxo internacional do comércio de fumo, indicando que o volume transacionado no mercado internacional de fumo foi menor na presença de tais medidas. Além disso, pelo resultado do modelo gravitacional estimado, verificou-se que esse efeito perdurou por períodos subsequentes à emissão das notificações. Já o coeficiente estimado para as medidas técnicas foi não significativo, indicando que essas medidas não afetam o comércio de fumo.

Dessa forma, dado o caráter restritivo das medidas sanitárias impostas, torna-se importante a inclusão de questões referentes à regulamentação dessas medidas nas discussões e processos de tomada de decisão das empresas exportadoras de fumo, uma vez que, caso não consigam se ajustar a tais exigências, o alto investimento necessário e o 
efeito duradouro dessas medidas podem inviabilizar seu acesso ao mercado internacional.

Em termos de ações governamentais, seria interessante a criação ou aperfeiçoamento de políticas no sentido de aumentar o caráter informativo das medidas sanitárias, principalmente aquelas que envolvem questões relacionadas à saúde humana e proteção da planta, de forma a reduzir o tempo que as empresas exportadoras utilizam para se adequar a tais exigências.

Por fim, sugere-se, para novos estudos, a desagregação das notificações (SPS e TBT) com base nos diferentes objetivos que essas medidas buscam atender, de forma a identificar qual exigência causa maior efeito sobre o fluxo do comércio de fumo.

\section{REFERÊNCIAS}

ALMEIDA, Fernanda Maria de. Efeitos dos custos de transporte e das barreiras comerciais no comércio internacional de café verde. 2009. 85p. Dissertação (Mestrado) - Universidade Federal de Viçosa, Viçosa, 2009.

ALVES, Greigiano José. Impacto de medidas não tarifárias na fruticultura brasileira. 2012. 116p. Dissertação (Mestrado) - Universidade Federal de Viçosa, Viçosa, 2012.

ALVES, G. J.; GOMES, M. F. M.; GONÇALVES, L. V. Análise do impacto da regulamentação SPS e TBT nas importações de uva in natura brasileira. $49^{\circ}$ Congresso da Sober, Belo Horizonte, MG, 2011, 15p.

ALVES, M. C. Identificação e efeitos das barreiras não tarifárias às exportações brasileiras de carne de frango. 2008. 150p. Dissertação (Mestrado) - Universidade Federal de Viçosa, Viçosa, 2008.

ANDERSON, J. Theoretical foundation for the gravity equation. American Economic Review, Nashville, v.69, n.1., 1979. Disponível em: $<$ http://ideas.repec.org/a/aea/aecrev/v69y1 979ip 106-16.html>. Acesso em maio de 2012. 
ANDERSON,J. WINCOOP, E. Gravity with gravitas: A solution to the border puzzle. American Economic Review, Nashville, v.93, n.1, 2003. Disponível em: <http://ideas.repec.org/p/boc/bocoec/485.html>. Acesso em: maio 2012.

AFUBRA - Associação dos Fumicultores do Brasil. Disponível em: $<$ www.afubra.com.br>. Acesso em: ago. 2015.

BALDWIN,J.; YOTOV, Y. The changing incidence of geography. Cambridge, 2008. (NBER Working Paper No. W14423). Disponível em: $<$ www.ssrn.com/abstract $=945443>$. Acesso em: maio 2012.

BALDWIN, R.E.; TAGLIOLI, D. Gravity for dummies and dummies for gravity equations. London, 2006. (CEPR Discussion Paper, 5850). Disponível em: <http://ssrn. com/abstract=945443 $>$. Acesso em: jun. 2012.

BERGSTRAND, J. The gravity equation in international trade: some microeconomic foundations and the empirical evidence. Review of Economics and Statistics, v.67, p. 474-481, 1985.

BURNQUIST, H. L; SOUZA, M. J. P. Impactos da regulamentação sanitária sobre o comércio: positivo, negativo ou ambíguo? In: Notificações aos Acordos de Barreiras Técnicas (TBT) e Sanitárias (SPS) da OMC: transparência comercial ou barreiras não tarifárias? Editor: Orlando $M$. da Silva, MG, 2010.

CEPII - Centre D'études prospectives et d'informations internationales. Databases \& models. Disponível em: <http://www.cepii. $\mathrm{fr} /$ anglaisgraph/bdd/bdd.htm $>$. Acesso em: maio 2012.

CHENG, C.; WALL, H. J. Controlling for Heterogenity in Gravity Models of Trade and Integration. Federal Reserve Bank of St. Louis Review, St. Louis, v.87, n. 1, 2005. 
DA MATA, D.F.G; FREITAS, R. E. Produtos agropecuários: para quem exportar? Revista de Economia e Sociologia Rural, v. 46, p.257-290. Brasília: SOBER, 2008.

DAMIÃO, Daiana Nogueira. Impactos dos instrumentos regulatórios SPS e TBT sobre o comércio de carne bovina dos países do Mercosul. 2011. 155p. Dissertação (Mestrado) - Universidade Federal de Viçosa, Viçosa, 2011.

DEARDOF, A. V. Determinants of bilateral trade: does gravity work in a neoclassical world? Cambridge, 1995. (NBER Working Paper No. W5377). Disponível em: <http://ssrn.com/abstract=93748>. Acesso em: maio 2012.

DISDIER, A.; FONTAGNÉ, L.; MIMOUNI, M. The impact of regulations on agricultural trade: evidence from the SPS and TBT agreements. American Journal of Agricultural economics, Oxford, v.90, n.2, p 1-7, 2008.

FASSARELLA, L. M.; SOUZA, M, J, P.; VALDES, C.; BURNQUIST, H. L. Regulamentações sanitárias e fitossanitárias e comércio das Américas. $48^{\circ}$ Congresso da Sober, Campo Grande, MS, 2010, 20p.

FEENSTRA, R. C. Advanced international trade: theory and evidence. Princeton: Princeton University Press, 2004, 484 p.

FEISTEL, P.R. A natureza do comércio das Regióes Brasileiras no Mercosul. Tese de doutorado, Departamento de Economia, UFPE, Recife, 2006

FONTAGNÉ, L.; von KIRCHBACH, F.; MIMOUNI, M. An assessment of environmentally related nontariff measures. World Economic, Hoboken, v.28, n.10, p. 1417-1439, 2005.

GREENE, W. H. Econometric analysis. 2. Ed, New York; Macmillan, 1993. $791 p$. 
INMETRO - Instituto Nacional de Metrologia, Normalização e Qualidade Industrial. Disponível em: <http://www.inmetro.gov.br/>. Acesso em: maio 2012.

GOURIEROUX, C.; MONFORT, A.; TROGNON, A. Pseudo Maximum Likehood methods: applications to Poisson models. Econometrica, n.52, p. 701-720, 1984.

GUJARATI, D. N. Econometria Básica. Editora Elsevier Brasil, $4^{\mathrm{a}}$ edição. Rio de Janeiro, Campus, 2006.

HEAD, K.; MAYER, T. Non-Europe: the Magnitude and causes of Market fragmentation in Europe. Weltwirstschaftliches Archiv, Vol. 136, no. 2, 2000.

MACMAP - Market Access Map. Disponível em: <www.macmap.org>. Acesso em: maio 2012.

MIRANDA, Silvia Helena Galvao de. Quantificação dos efeitos das barreiras não-tarifárias sobre as exportações brasileiras de carne bovina. 2001. 233f. Tese (Doutorado em Economia Aplicada) - Escola Superior de Agricultura Luiz de Queiroz, Piracicaba, 2001.

OTSUKI, T.; WILSON, J. S; SEWADEH, M. What price precaution? European harmonization of afloatoxin regualations and African groundnut exports. European Review of Agricultural Economics, v.28, n.3, p.263283, 2001.

PINDYCK, R. S. ; RUBINFELD, D. L. Microeconomia. Editora Pearson Education, $6^{\text {a }}$ edição, 2005.

ROBERTS, D.; ORDEN, D.; JOSLING, T. A framework for analyzing technical barriers to agricultural markets. Washington (DC): U.S Departament of Agricultural, Economic Research Service, 1999; 52p. (Technical Bulletin, 1876) 
SALVATORE, D. Economia internacional. Rio de Janeiro: Livros Técnicos Científicos (LTC), 1999.

SANTOS SILVA, J.M.C.; TENREYRO, S. The log of gravity. The Review of Economics and Statistics, Cambridge, v. 88, n. 4, 2006.

SCHLUETER, S. W.; WIECK, C.; HECKELEI, T. Regulatory SPS instruments in meat trade. Germany, 2009 (IATRC Discussion Paper 2009). Disponível em:

$<$ http://iatrc.software.umn.edu/activities/annualmeetings/themedays/p dfs/2009Dec-Wieck.pdf>. Acesso em: jun. 2012.

SECRETARIA DE COMÉRCIO EXTERIOR (SECEX). Disponível em: $<$ www.mdic.gov.br $>$. Acesso: em jul. 2015.

SHEPHERD, B.; WILSON, J.S. Trade facilitation in ASEAN member countries: measuring progress and assessing priorities. Washington: World Bank, 2008. (Working Paper, 4615).

SILIVERSTOVS, B.; SCHUMACHER, D. Estimating gravity equations: to log or not tolog? Berlin, 2007. 42 p. (Discussion Papers of DIW Berlin, 739). Disponível em:

$<$ http://ideas.repec.org/p/diw/diwwpp/dp739.html $>$. Acesso em junho de 2012.

SINDITABACO - Sindicato da Indústria do Tabaco. Disponível em: $<$ www.sinditabaco.com.br $>$. Acesso em: ago. 2015.

SILVA, O.; ALMEIDA, F., M. A incidência das notificações os acordos sobre medidas SPS e TBT da OMC nas exportações agrícolas do Brasil. In: Notificações aos Acordos de Barreiras Técnicas (TBT) e Sanitárias (SPS) da OMC: transparência comercial ou barreiras não tarifárias? Editor: Orlando M. da Silva, MG, 2010, 239p.

SILVA, F. A.; LÍRIO, V. S.; CORONEL, D. A.; GOMES, M. F. M. Identificação e efeitos de medidas não tarifárias impostas aos principais exportadores 
de carne suína. Revista de Economia Mackenzie, v.9, n. 3, p. 34-52, 2011.

SWANN, G.M.P.; TEMPLE, P. ; SHURMER, M. Standards and trade performance: the British experience. Economic Journal, Malden, v. 106, n. 438, p. 1297-1313, 1996.

THILMANY, D.D; BARRET, C.B (1997). Regulatory barriers in an integrating world food market. Review of Agricultural Economics. Volume 19, number 1. PP 91-107.

UNCOMTRADE - Sistem United Nations Commodity Trade. Disponível em: <http://comtrade.un.org/>. Acesso em: maio 2012.

UNCTAD. Training package on trade policy analysis, Module 2. EX-post Statistical Analysis. [s.I.;s.n.], 2008.

Submetido em 12/07/2013.

Aprovado em $02 / 07 / 2015$.

\section{Sobre os autores}

\section{Carlos Otávio de Freitas}

Mestre em Economia Aplicada pela Universidade Federal de Viçosa - UFV. Doutorando em Economia Aplicada do Departamento de Economia Rural (DER) pela Universidade Federal de Viçosa - UFV.

Endereço: Rua Marechal Dedoro, 140, Bairro: Palmeiras. 35430-231 - Ponte Nova, MG, Brasil.

E-mail: carlos.freitas@ufv.br

\section{Marília Fernandes Maciel Gomes}

Doutora em Economia Rural, Professora Associada da Universidade Federal de Viçosa. E-mail:mfmgomes@ufv.br

\section{Fernanda Maria de Almeida}

Doutora em Economia Aplicada, Professora Adjunta, Departamento de Administração, Universidade Federal de Viçosa.

E-mail: Fernanda.almeida@ufv.br

Fernanda Aparecida Silva

Mestranda em Economia Aplicada, Universidade Federal de Viçosa. 
As medidas aplicadas aos acordos SPS e TBT sobre as exportações brasileiras de fumo

E-mail: fernanda.aparecida@ufv.br 